\title{
Critical indices from self-similar root approximants
}

\author{
S. Gluzman and V.I. Yukalov ${ }^{a}$ \\ Bogolubov Laboratory of Theoretical Physics, \\ Joint Institute for Nuclear Research, Dubna 141980, Russia
}

\begin{abstract}
The method of self-similar root approximants has earlier been shown to provide accurate interpolating formulas for functions for which small-variable expansions are given and the behaviour of the functions at large variables is known. Now this method is generalized for the purpose of extrapolating small-variable expansions to the region of finite and large variables, where the sought function exhibits critical behaviour. The procedure of calculating critical indices is formulated and illustrated by a variety of physical problems.
\end{abstract}

${ }^{a}$ Corresponding author: V.I. Yukalov

E-mail: yukalov@theor.jinr.ru 


\section{Formulation of problem}

A real function $f(x)$ of a real variable $x$ is said to exhibit critical behaviour, with a critical index $\beta$, at a finite critical point $x_{c}$, when in the vicinity of this point it behaves as

$$
f(x) \simeq B\left(x_{c}-x\right)^{\beta} \quad\left(x \rightarrow x_{c}-0\right) .
$$

The function can tend to infinity, if the critical index $\beta$ is negative, or to zero, if this index is positive.

The critical behaviour can also occur at infinity, where the function behaves as

$$
f(x) \simeq B x^{\beta} \quad(x \rightarrow \infty),
$$

with the critical index $\beta$. Respectively, the function can tend to infinity, if $\beta$ is positive and to zero, if $\beta$ is negative. The critical behaviour at infinity can formally be interpreted as the case, where the critical point is located at infinity.

Critical phenomena are widespread in physics. And it is an important problem of defining the related critical indices. However, for realistic physical systems it is often impossible to get exact solutions, but the sole thing one can do is to resort to perturbation theory for obtaining the behavior of the sought function at small variable,

$$
f(x) \simeq f_{k}(x) \quad(x \rightarrow 0)
$$

where the function is approximated by an expansion

$$
f_{k}(x)=f_{0}(x)\left(1+\sum_{n=1}^{k} a_{n} x^{n}\right)
$$

with $f_{0}(x)$ being a known factor. Without loss of generality, we may assume that this prefactor has the form

$$
f_{0}(x)=A x^{\alpha}
$$

Such expansions are usually asymptotic and strongly divergent not allowing for their use at finite values of the variable.

In order to understand whether the function possesses critical behaviour, it is, first of all, necessary to extrapolate the asymptotic expansion (4) to finite and even large values of the variable. Such an extrapolation can be accomplished by means of Padé and Borel summation or other techniques usually requiring the knowledge of many expansion terms [1,2]. In addition, these summation techniques not always are applicable, as is discussed in Refs. [3, 4, How would it be possible to obtain reliable results for the critical indices employing a small number of terms in the asymptotic expansion?

An efficient method of summation of divergent series has been developed in the frame of self-similar approximation theory [5]. A variant of this method, called self-similar root approximants, has been shown by a number of problems [9-14] to provide accurate interpolation formulae, when both the small-variable expansion and the large-variable behaviour are known. The accuracy of such an interpolation was demonstrated to be not worse and in many cases better than that of two-point Padé approximants, when these could be defined.

In the present paper, we generalize the method of self-similar root approximants for the extrapolation purpose. We consider the situation, when only small-variable expansions are given, 
and one needs to extrapolate such expansions to finite, or even infinite, values of the variable, not knowing the large-variable behaviour of the sought functions. The main attention will be payed to the problem of calculating the critical indices.

According to the general theory [11 14], a self-similar root approximant, based on the smallvariable expansion (4), has the form

$$
\frac{f_{k}^{*}\left(x, n_{k}\right)}{f_{0}(x)}=\left(\left(\left(1+A_{1} x\right)^{n_{1}}+A_{2} x^{2}\right)^{n_{2}}+\ldots+A_{k} x^{k}\right)^{n_{k}}
$$

in which all parameters $A_{j}$ are found from the comparison of the like orders of the re-expansion of equation (6) in powers of $x$ with the given expansion (44). This method of equating the like powers of $x$ is sometimes called the accuracy-through-order procedure. The internal powers are defined as

$$
n_{j}=\frac{j+1}{j} \quad(j=1,2, \ldots, k-1),
$$

while the external power $n_{k}$ plays the role of a control function to be determined from additional conditions.

If the large-variable power $\beta$ in equation (2) were known, then we could compare the latter equation with the large-variable behaviour of the root approximant (므) being

$$
f_{k}^{*}\left(x, n_{k}\right) \simeq B_{k} x^{\alpha+k n_{k}},
$$

where $\alpha$ is introduced in equation (5) and

$$
B_{k}=A\left(\left(\left(A_{1}^{n_{1}}+A_{2}\right)^{n_{2}}+A_{3}\right)^{n_{3}}+\ldots+A_{k}\right)^{n_{k}} .
$$

This comparison yields the relation

$$
\alpha+k n_{k}=\beta
$$

defining the external power

$$
n_{k}=\frac{\beta-\alpha}{k},
$$

provided $\beta$ is known. This way of defining the external power has been used in our previous papers on the application of the root approximants as interpolating formulae. When several terms in the large-variable behaviour are known, then the related powers prescribe the values for the corresponding number of external powers $n_{j}$.

But now we consider the situation where the large-variable behaviour of the function is not known, hence $\beta$ is not given. Moreover, the critical behaviour can happen at a finite value $x_{c}$ of the variable $x$. The development of the method for defining the critical index $\beta$ by employing self-similar root approximants is the aim of the present paper.

\section{Method of defining critical indices}

Suppose we can construct several root approximants $f_{k}^{*}\left(x, n_{k}\right)$, in which the external power $n_{k}$ plays the role of a control function. Following the idea of self-similar approximation theory [5-7], it is possible to treat the sequence $\left\{f_{k}^{*}\left(x, n_{k}\right)\right\}$ as a trajectory of a dynamical system, with the approximation order $k$ playing the role of discrete time. A discrete-time dynamical system is 
called cascade. Here it is termed the approximation cascade, since its trajectory consists of the sequence of approximants. The cascade velocity is defined by the Euler discretization formula

$$
V_{k}\left(x, n_{k}\right)=f_{k+1}^{*}\left(x, n_{k}\right)-f_{k}^{*}\left(x, n_{k}\right)+\left(n_{k+1}-n_{k}\right) \frac{\partial}{\partial n_{k}} f_{k}^{*}\left(x, n_{k}\right) .
$$

The effective limit of the sequence $\left\{f_{k}^{*}\left(x, n_{k}\right)\right\}$ corresponds to the fixed point of the cascade, where the cascade velocity tends to zero, as $k$ tends to infinity. Having a finite number of approximants, the cascade velocity is not necessarily tending to zero, but certainly has to diminish for the sequence being convergent. Thus, the control functions $n_{k}=n_{k}(x)$, controlling convergence, are defined as the minimizers of the absolute value of the cascade velocity [5] 8]:

$$
\left|V_{k}\left(x, n_{k}(x)\right)\right|=\min _{n_{k}}\left|V_{k}\left(x, n_{k}\right)\right| .
$$

A finite critical point $x_{k}^{c}$, in the $k$-th approximation, exists if the equation

$$
\left[f_{k}^{*}\left(x_{k}^{c}, n_{k}\right)\right]^{1 / n_{k}}=0 \quad\left(0<x_{k}^{c}<\infty\right)
$$

enjoys a finite solution for $x_{k}^{c}=x_{k}^{c}\left(n_{k}\right)$. Then the critical index in the $k$-th approximation is

$$
\beta_{k}=\lim _{x \rightarrow x_{k}^{c}} n_{k}(x) \quad\left(0<x_{k}^{c}<\infty\right)
$$

When we are studying the critical behaviour at infinity, which we denote as $x_{c} \sim \infty$, keeping in mind that this case formally corresponds to the critical point at infinity, then the critical index is

$$
\beta_{k}=\alpha+k \lim _{x \rightarrow \infty} n_{k}(x) \quad\left(x_{c} \sim \infty\right),
$$

where $\alpha$ is defined in equation (5).

Thus the critical indices are defined, provided the control functions $n_{k}(x)$ are found. However, the minimization of the cascade velocity (12) poses some problems. First of all, equation (13) contains two control functions, $n_{k+1}$ and $n_{k}$. Hence it is impossible to find two solutions from one equation. But it is possible to simplify the problem, when minimizing velocity (12), so that to get one equation for one control function. This is admissible to accomplish in two ways.

For instance, keeping in mind that $n_{k+1}$ is close to $n_{k}$, equation (13) reduces to the minimal difference condition

$$
\min _{n_{k}}\left|f_{k+1}^{*}\left(x, n_{k}\right)-f_{k}^{*}\left(x, n_{k}\right)\right| \quad(k=1,2, \ldots) .
$$

In particular, one can look for a solution $n_{k}=n_{k}(x)$ of the equation

$$
f_{k+1}^{*}\left(x, n_{k}\right)-f_{k}^{*}\left(x, n_{k}\right)=0
$$

If the latter does not possess a solution for $n_{k}$, one has to return to form (17)).

In general, when nothing is known on the form of the sought function $f(x)$, the control functions $n_{k}$, being the solutions of equation (18), depend on the variable $x$. But when we are looking for a function $f(x)$ in the vicinity of its critical point $x_{c}$, where the function $f(x)$ acquires form (11), the control functions are to be treated as the limits of $n_{k}(x)$ for $x \rightarrow x_{c}$. So that the control functions $n_{k}$, characterizing the critical behaviour of $f(x)$ near a critical point $x_{c}$, become the numbers $n_{k}\left(x_{c}\right)$. In what follows, writing for short $n_{k}$, we assume $n_{k}=n_{k}\left(x_{c}\right)$. 
In the vicinity of a finite critical point, the function $f_{k}^{*}$ behaves as

$$
f_{k}^{*}\left(x, n_{k}\right) \simeq f_{0}(x)\left(1-\frac{x}{x_{k}^{c}}\right)^{n_{k}} \quad\left(x \rightarrow x_{k}^{c}-0\right) .
$$

Then condition (18) becomes

$$
x_{k+1}^{c}\left(n_{k}\right)-x_{k}^{c}\left(n_{k}\right)=0 \quad\left(0<x_{k}^{c}<\infty\right) .
$$

When the critical behaviour occurs at infinity, then it is convenient to introduce the control function

$$
s_{k} \equiv k n_{k},
$$

so that the large-variable behaviour of the root approximants reads as

$$
f_{k}^{*}\left(x, s_{k}\right) \simeq B_{k}\left(s_{k}\right) x^{\alpha+s_{k}} \quad(x \rightarrow \infty) .
$$

As a result, the minimal difference condition

$$
f_{k+1}^{*}\left(x, s_{k}\right)-f_{k}^{*}\left(x, s_{k}\right)=0
$$

leads to the equation

$$
B_{k+1}\left(s_{k}\right)-B_{k}\left(s_{k}\right)=0 \quad\left(x_{k}^{c} \sim \infty\right) .
$$

The other equation for defining control functions follows from the minimal velocity condition (13) by keeping in mind that $f_{k+1}^{*}$ is close to $f_{k}^{*}$ and usually $n_{k+1}$ does not exactly coincide with $n_{k}$, because of which one has to consider the minimal derivative condition

$$
\min _{k}\left|\frac{\partial}{\partial n_{k}} f_{k}^{*}\left(x, n_{k}\right)\right| \quad(k=1,2, \ldots) .
$$

In particular, one can look for the solution of the equation

$$
\frac{\partial}{\partial n_{k}} f_{k}^{*}\left(x, n_{k}\right)=0
$$

However, the minimal derivative condition cannot be applied directly, when the sought function exhibits critical behaviour, where the function either diverges or tends to zero. To apply this condition, it is necessary to extract from the function nondivergent parts. For example, if the critical point is finite, one can study the residue of the function $\partial \ln f_{k}^{*} / \partial n_{k}$, for which we have

$$
\lim _{x \rightarrow x_{k}^{c}}\left(x_{k}^{c}-x\right) \frac{\partial}{\partial n_{k}} \ln f_{k}^{*}\left(x, n_{k}\right)=n_{k} \frac{\partial x_{k}^{c}}{\partial n_{k}} .
$$

Therefore, instead of equation (26), we get the condition

$$
\frac{\partial x_{k}^{c}}{\partial n_{k}}=0 \quad\left(0<x_{k}^{c}<\infty\right)
$$

And when the critical behaviour occurs at infinity, then we can consider the limit

$$
\lim _{x \rightarrow \infty} \frac{f_{k}^{*}\left(x, s_{k}\right)}{x^{\alpha+s_{k}}}=B_{k}\left(s_{k}\right),
$$


for which equation (26), defining control functions, reduces to the form

$$
\frac{\partial B_{k}\left(s_{k}\right)}{\partial s_{k}}=0 \quad\left(x_{k}^{c} \sim \infty\right)
$$

To better explain the suggested techniques, let us consider a simple example, when the sought function leads to the small-variable expansion

$$
f(x) \simeq 1+a_{1} x+a_{2} x^{2} \quad(x \rightarrow 0) .
$$

The first-order root approximant is

$$
f_{1}^{*}\left(x, n_{1}\right)=(1+A x)^{n_{1}}
$$

with

$$
A=A\left(n_{1}\right)=\frac{a_{1}}{n_{1}}
$$

found from the accuracy-through-order procedure. Note that this form (30) reminds us the Sommerfeld formula used in nuclear physics [15]. Expression (30) possesses a finite critical point, provided there exists a finite positive value

$$
x_{1}^{c}\left(n_{1}\right)=-\frac{1}{A\left(n_{1}\right)}=-\frac{n_{1}}{a_{1}} .
$$

The second-order root approximant reads as

$$
f_{2}^{*}\left(x, n_{2}\right)=\left(\left(1+A_{1} x\right)^{2}+A_{2} x^{2}\right)^{n_{2}},
$$

with the parameters

$$
A_{1}=A_{1}\left(n_{2}\right)=\frac{a_{1}}{2 n_{2}}, \quad A_{2}=A_{2}\left(n_{2}\right)=\frac{a_{1}^{2}\left(1-2 n_{2}\right)+4 a_{2} n_{2}}{4 n_{2}^{2}}
$$

Now the finite critical point is given by a positive value

$$
x_{2}^{c}\left(n_{2}\right)=\frac{-A_{1}\left(n_{2}\right) \pm \sqrt{-A_{1}\left(n_{2}\right)}}{A_{1}^{2}\left(n_{2}\right)+A_{2}\left(n_{2}\right)} .
$$

The minimal difference condition (18), in the form

$$
f_{2}^{*}\left(x, n_{1}\right)-f_{1}^{*}\left(x, n_{1}\right)=0,
$$

is equivalent to the condition

$$
x_{2}^{c}\left(n_{1}\right)-x_{1}^{c}\left(n_{1}\right)=0
$$

which yields

$$
n_{1}=\frac{a_{1}^{2}}{a_{1}^{2}-2 a_{2}} .
$$

Hence the first-order critical point is

$$
x_{1}^{c}\left(n_{1}\right)=-\frac{n_{1}}{a_{1}}=\frac{a_{1}}{2 a_{2}-a_{1}^{2}} .
$$


In what follows, keeping in mind the minimal difference condition, we shall write, for simplicity, $x_{1}^{c}\left(n_{1}\right)=x_{2}^{c}\left(n_{1}\right) \equiv x_{c}$.

The corresponding root approximant (30) acquires the form

$$
f_{1}^{*}(x)=\left(1-\frac{x}{x_{c}}\right)^{\beta_{1}} \quad\left(0 \leq x \leq x_{c}\right)
$$

with the first-order critical index

$$
\beta_{1}=n_{1}=\frac{a_{1}^{2}}{a_{1}^{2}-2 a_{2}} .
$$

The second-order critical index $\beta_{2}=n_{2}$ is defined by the condition

$$
\frac{\partial}{\partial n_{2}} x_{2}^{c}\left(n_{2}\right)=0
$$

When there is no finite critical point, but the critical behaviour happens at infinity, then we have to consider the large-variable asymptotic forms for the root approximants, with the substitution (21). The first-order approximant gives

$$
f_{1}^{*}\left(x, s_{1}\right) \simeq B_{1}\left(s_{1}\right) x^{s_{1}} \quad(x \rightarrow \infty),
$$

where

$$
B_{1}\left(s_{1}\right)=A\left(s_{1}\right)^{s_{1}} .
$$

While the second-order approximant leads to

$$
f_{2}^{*}\left(x, s_{2}\right) \simeq B_{2}\left(s_{2}\right) x^{s_{2}} \quad(x \rightarrow \infty)
$$

with

$$
B_{2}\left(s_{2}\right)=\left[A_{1}\left(s_{2}\right)^{2}+A_{2}\left(s_{2}\right)\right]^{s_{2} / 2} .
$$

The first-order critical index $\beta_{1}=s_{1}$ is found form the condition

$$
B_{2}\left(s_{1}\right)-B_{1}\left(s_{1}\right)=0 \quad\left(\beta_{1}=s_{1}\right) .
$$

And the second-order critical index $\beta_{2}=s_{2}$ can be obtained form the condition

$$
\frac{\partial B_{2}\left(s_{2}\right)}{\partial s_{2}}=0 \quad\left(\beta_{2}=s_{2}\right)
$$

The final answer can be presented as the average

$$
\beta^{*}=\frac{1}{2}\left(\beta_{1}+\beta_{2}\right) \pm \frac{1}{2}\left|\beta_{1}-\beta_{2}\right| .
$$

In the similar way, one can proceed to higher orders. However, our aim here is to show that even in the lower orders we get rather accurate critical indices, which is demonstrated for various problems in Secs. 4 to 12. The existence of numerical convergence for higher orders is illustrated in Sec. 14. Section 15 concludes. 


\section{Comparison with method of Padé approximants}

Asymptotic series are often approximated by Padé approximants. The latter is denoted as $P_{M / N}(x)$ implying the ratio of a polynomial of order $M$ with respect to $x$ to a polynomial of order $N$. The coefficients of the polynomials are defined by comparing the like orders of expansions in powers of $x$ of the Padé approximant and of the sought function $f_{k}(x)$. To emphasize this fact, one often denotes the related Padé approximant as $P_{M / N}(x, f)$. These approximants provide the best approximation in the class of rational functions [1]. However the functions in the vicinity of their critical points, in general, are non-rational. Therefore the direct use of Padé approximants for functions exhibiting critical behaviour is impossible. Really, a Padé approximant $P_{M / N}$ can have a pole that could be associated with a finite critical point, but the related critical index would be an integer, while usually critical indices are not integers. The same concerns the large-variable behaviour of $P_{M / N}(x)$, where the power of $x$ is always an integer $M-N$.

The problem can be circumvented by means of the Dlog-Padé method [1,16]. Then, instead of the function $f(x)$, one considers the function

$$
g(x) \equiv \frac{d}{d x} \ln f(x) .
$$

Near a finite critical point $x_{c}$, where the function $f(x)$ is of the form (11), function (46) behaves as

$$
g(x) \simeq \frac{\beta}{x-x_{c}} \quad\left(x \rightarrow x_{c}-0\right) .
$$

Hence the pole here defines the critical point $x_{c}$, while the critical index is given by the residue

$$
\beta=\lim _{x \rightarrow x_{c}}\left(x-x_{c}\right) g(x) .
$$

When the sought function is represented by expansion (44), function (46) takes the form

$$
\frac{d}{d x} \ln f_{k}(x)=\frac{\alpha}{x}+g_{k}(x),
$$

in which the second term can be expanded in powers of $x$ yielding

$$
g_{k}(x) \simeq \sum_{n=0}^{k} a_{n}^{\prime} x^{n} \quad(x \rightarrow 0),
$$

with the coefficients $a_{n}^{\prime}$ expressed through $a_{n}$. The finite series (50) can be extrapolated by Padé approximants $P_{M / N}(x, g)$, with $M+N \leq k$. The pole nearest to zero of the latter approximant defines the critical point $x_{c}$, and the critical index is given by the residue

$$
\beta_{M / N}=\lim _{x \rightarrow x_{c}}\left(x-x_{c}\right) P_{M / N}(x, g) .
$$

For a function $f(x)$, with the critical behaviour at infinity, where it has form (2), function (46) behaves as

$$
g(x) \simeq \frac{\beta}{x} \quad(x \rightarrow \infty) .
$$

This implies that the critical index can be obtained from the limit

$$
\beta=\lim _{x \rightarrow \infty} x g(x) .
$$


It is clear that extrapolating the function $g_{k}(x)$ by Padé approximants $P_{M / N}(x, g)$, not all $M$ and $N$ are permitted, since in the large-variable limit the Padé approximants $P_{M / N}(x, g)$ exhibit different behaviour depending on the relation between $M$ and $N$ :

$$
\lim _{x \rightarrow \infty} P_{M / N}(x, g)= \begin{cases}0, & M<N \\ \text { const }, & M=N \\ \infty, & M>N\end{cases}
$$

In order that the critical index (53) be finite, it is necessary to take the approximants $P_{N / N+1}(x, g)$, so that the corresponding approximation

$$
\beta_{N / N+1}(x, g)=\lim _{x \rightarrow \infty} x P_{N / N+1}(x, g)
$$

be finite.

As is known [1], for a given expansion of order $k$, one can construct the whole table of Padé approximants. This means that defining the critical indices through the Dlog-Padé method is not a uniquely defined procedure. And different Padé approximants can result in basically different values. Then it is not clear which of these quantities to prefer.

For illustration, let us again consider the case of expansion (29). We define function (50) and construct the related Padé approximants $P_{M / N}(x, g)$, with $M+N \leq 2$. The simplest Padé approximant here is

$$
P_{0 / 1}(x, g)=\frac{b_{0}}{1+b_{1} x},
$$

with the parameters

$$
b_{0}=a_{1}, \quad b_{1}=\frac{a_{1}^{2}-2 a_{2}}{a_{1}} .
$$

The pole of approximant (55) yields the critical point

$$
x_{c}=-\frac{1}{b_{1}}=\frac{a_{1}}{2 a_{2}-a_{1}^{2}} .
$$

The critical index

$$
\beta_{0 / 1}=\lim _{x \rightarrow x_{c}}\left(x-x_{c}\right) P_{0 / 1}(x, g)
$$

becomes

$$
\beta_{0 / 1}=\frac{b_{0}}{b_{1}}=\frac{a_{1}^{2}}{a_{1}^{2}-2 a_{2}} .
$$

In this case, comparing equations (56) with (37) and (57) with (39), we notice that the simplest Dlog-Padé approximation $P_{0 / 1}$ gives the critical point and critical index coinciding with those of the first root approximation. However, in the frame of the Dlog-Padé method, the choice of a particular Padé approximant is not uniquely defined, since there are several possibilities of choosing such Padé approximants. Thus, we can take

$$
P_{1 / 1}(x, g)=\frac{c_{0}+c_{1} x}{1+c_{2} x},
$$

with the parameters

$$
c_{0}=a_{1}, \quad c_{1}=\frac{4 a_{2}-a_{1}^{2}}{2 a_{2}-a_{1}^{2}} a_{2}, \quad c_{2}=\frac{3 a_{2}-a_{1}^{2}}{2 a_{2}-a_{1}^{2}} a_{1} .
$$


Then the critical point is

$$
x_{c}^{\prime}=-\frac{1}{c_{2}}=-\frac{2 a_{2}-a_{1}^{2}}{a_{1}\left(3 a_{2}-a_{1}^{2}\right)} .
$$

And the critical index

$$
\beta_{1 / 1}=\lim _{x \rightarrow x_{c}^{\prime}}\left(x-x_{c}^{\prime}\right) P_{1 / 1}(x, g)
$$

becomes

$$
\beta_{1 / 1}=\frac{a_{1}^{2}\left(4 a_{2}-a_{1}^{2}\right)-4 a_{2}^{2}}{2 a_{2}-a_{1}} .
$$

These values can be quite different from the previously found. The critical points (59) and (56) are connected by the relation

$$
x_{c}^{\prime}=-\frac{1}{\left(3 a_{2}-a_{1}^{2}\right) x_{c}},
$$

while the critical indices (60) and (57), by the relation

$$
\beta_{1 / 1}=\left(2 a_{2}-a_{1}^{2}\right)^{2} \frac{\beta_{0 / 1}}{a_{1}^{2}} .
$$

As is seen from equality (61), under the condition $3 a_{2}>a_{1}^{2}$, the critical points $x_{c}$ and $x_{c}^{\prime}$ are of different signs. Hence, if one of them is positive, the other is negative, that is, does not exist as a physically acceptable solution. And the critical indices $\beta_{1 / 1}$ and $\beta_{0 / 1}$ can be quite different. This is contrary to the case of root approximants, where the second-order approximations for the critical point (33) and the critical index (40) do not contradict the first-order values.

In the following sections, by treating a number of concrete examples, we show that the DlogPadé approximation, based on approximant (58), either does not possess physical solutions or is worse than the values given by the root approximants. Moreover, analyzing the numerical convergence of solutions in Sec. 14, we demonstrate that the root approximants yield a sequence of solutions converging to the exact value of a critical index, while the sequence, based on the Dlog-Padé method, is divergent and results in unphysical solutions.

\section{Susceptibility of two-dimensional Ising model}

Consider the two-dimensional Ising model characterized by the Hamiltonian

$$
\hat{H}=-\frac{J}{2} \sum_{\langle i j\rangle} s_{i}^{z} s_{j}^{z} \quad\left(s_{j}^{z} \equiv \frac{S_{j}^{z}}{S}\right)
$$

on a square lattice, with the ferromagnetic interaction of nearest neighbours, for spins $S_{j}^{z}= \pm 1 / 2$. The dimensionless interaction parameter is defined as

$$
g \equiv \frac{J}{k_{B} T} .
$$

The susceptibility is known [17] to diverge at a critical point

$$
g_{c}=\frac{1}{2} \ln (1+\sqrt{2})=0.440687
$$


as

$$
\chi(g) \propto\left(g_{c}-g\right)^{-\gamma},
$$

with the critical index $\gamma=7 / 4$. The weak-interaction or high-temperature expansion of the susceptibility yields [18] the series in powers of $g$,

$$
\chi(g) \simeq 1+4 g+12 g^{2} \quad(g \rightarrow 0) .
$$

Employing the method of Sec. 2, we find the critical point $g_{c}=0.5$. The first-order approximation for the critical index $\gamma_{1}=2$ differs from the exact one by $14.29 \%$ and the second-order approximation $\gamma_{2}=1.846$ is accurate within $5.49 \%$. Then the value of the index is estimated as

$$
\gamma^{*}=1.923 \pm 0.077 .
$$

Using high-temperature expansions for susceptibility [18], we have also calculated the critical indices $\gamma$ for the three-dimensional Ising model with different spins $S=1 / 2,3 / 2,5 / 2,7 / 2,1,2$, $3,4,5, \infty$. Since the critical index should not depend on the spin value, we averaged the results for different spins obtaining $\gamma^{*}=1.2396 \pm 0.0621$, which is close to the index $\gamma=1.234 \pm 0.005$ found by other sophisticated methods [19]. Note that the technique of [8] was employed for calculations for each spin separately.

The Dlog-Padé method with the approximant $P_{1 / 1}$, given in equation (58), does not have physical solutions.

\section{$5 \quad$ Effective viscosity of hard-sphere suspensions}

The problem of perfectly rigid spherical inclusions randomly embedded into an incompressible matrix is analogous to the problem of high-frequency effective viscosity of a hard-sphere suspension [20 22]. The viscosity, considered as a function of the variable

$$
\varphi \equiv \frac{4 \pi}{3} r_{s}^{3} \rho \quad\left(\rho \equiv \frac{N}{V}\right),
$$

in which $r_{s}$ is the sphere radius and $\rho$ is average density, exhibits the critical behaviour

$$
\eta(\varphi) \propto\left(\varphi_{c}-\varphi\right)^{-\mu} \quad\left(\varphi \rightarrow \varphi_{c}-0\right),
$$

where [23]

$$
\varphi_{c}=0.637, \quad \mu=1.7 .
$$

The small $\varphi$-expansion reads as

$$
\eta(\varphi) \simeq 1+\frac{5}{2} \varphi+5.0022 \varphi^{2} \quad(\varphi \rightarrow 0) .
$$

Using our method, we find the critical point $\varphi_{c}=0.666$. The critical index is $\mu_{1}=1.665$, with the error $2 \%$ and $\mu_{2}=1.788$, with the error $5 \%$. So the answer is

$$
\mu^{*}=1.726 \pm 0.06
$$

The Dlog-Padé method, with the approximant $P_{1 / 1}$, again does not provide physical solutions. 


\section{Conductivity in two-dimensional site percolation}

The problem of site percolation conductivity is studied within the framework of a minimal model for transport of classical particles through a random medium [24]. This minimal model, known as the Lorenz 2D gas, is a particularly simple statistical hopping model allowing both for analytical consideration and numerical simulations [24,25]. It can be realized on a square lattice with a fraction of sites being excluded at random. The test particle, or tracer, walks randomly with Poisson-distributed waiting times between the moves. At every move the tracer attempts to jump on to one of the neighboring sites also selected at random. The move is accepted if the site is not excluded. Through the diffusion coefficient for the tracer one can express the macroscopic conductivity [24]. The diffusion ceases to exist at the critical density of the excluded sites. If $f$ stands for the concentration of conducting or not excluded sites in the Lorenz model, then $x=1-f$ is the concentration of excluded sites. In the vicinity of the site percolation threshold [26] the conductivity behaves as

$$
\sigma(x) \propto\left(x_{c}-x\right)^{t} \quad\left(x \rightarrow x_{c}-0\right),
$$

with

$$
x_{c}=0.4073, \quad t=1.310 .
$$

Perturbation theory in powers of the variable $x=1-f$ gives [24] for the two-dimensional square lattice the expansion

$$
\sigma(x) \simeq 1-\pi x+1.28588 x^{2} \quad(x \rightarrow 0) .
$$

In our approach, we obtain the percolation threshold $x_{c}=0.4305$ and the critical index $t_{1}=1.352$, with an error $3 \%$ and $t_{2}=1.423$, with an error $8.6 \%$. The final answer is

$$
t^{*}=1.388 \pm 0.036 \text {. }
$$

The Dlog-Padé method, with the approximant $P_{1 / 1}$, gives $t=1.0896$, which is worse than the above approximation, differing from the exact value by $17 \%$.

\section{Conductivity in three-dimensional site percolation}

The three-dimensional problem, similar to the two-dimensional one, treated in the previous section, exhibits the critical behaviour [27 29] as in equation (71), with

$$
x_{c}=0.688, \quad t=1.9 .
$$

Perturbation theory gives

$$
\sigma(x) \simeq 1-2.52 x+1.52 x^{2} \quad(x \rightarrow 0) .
$$

Using our method, we get the critical point $x_{c}=0.761$. And for the critical index we have $t_{1}=1.918$, with an error $0.9 \%$ and $t_{2}=1.855$, with an error $2 \%$. The answer is

$$
t^{*}=1.887 \pm 0.032 .
$$

The Dlog-Padé method, based on the approximant $P_{1 / 1}$, yields $t=1.782$, with the error of $6 \%$, which is worse than the above value. 


\section{Permeability of sinusoidal two-dimensional channel}

Let us consider the widely studied case of the two-dimensional channel bounded by the surfaces

$$
z= \pm b(1+\varepsilon \cos x)
$$

where $\varepsilon$ is termed waviness. The permeability possesses the critical behaviour [30], when (in the case of $b=0.5)$ it tends to zero as

$$
K(\varepsilon) \simeq 0.100035\left(\varepsilon_{c}-\varepsilon\right)^{t} \quad\left(\varepsilon \rightarrow \varepsilon_{c}-0\right),
$$

with

$$
\varepsilon_{c}=1, \quad t=\frac{5}{2} .
$$

An expression for permeability as a function of the waviness parameter can be derived by perturbation theory in the form of an expansion in powers of the waviness [30, 31]. Thus, the permeability, for $b=0.5$, has the expansion

$$
K(\varepsilon) \simeq 1-3.14963 \varepsilon^{2}+4.08109 \varepsilon^{4} \quad(\varepsilon \rightarrow 0) .
$$

With our method, we find $\varepsilon_{c}=0.833$. For the critical index, we get $t_{1}=2.184$, with an error $12.6 \%$ and $t_{2}=2.559$, with an error $2.37 \%$. Thus we have

$$
t^{*}=2.372 \pm 0.19
$$

The Dlog-Padé method, with the approximant $P_{1 / 1}$, results in $t=1.884$, whose error is $25 \%$, which is less accurate than the above value.

\section{Ground-state energy of harmonium atom}

An $N$-electron harmonium atom is described by the Hamiltonian

$$
\hat{H}=\frac{1}{2} \sum_{i=1}^{N}\left(-\nabla_{i}^{2}+\omega^{2} r_{i}^{2}\right)+\frac{1}{2} \sum_{i \neq j}^{N} \frac{1}{r_{i j}},
$$

where dimensionless variables are used and

$$
r_{i} \equiv\left|\mathbf{r}_{i}\right|, \quad r_{i j} \equiv\left|\mathbf{r}_{i}-\mathbf{r}_{j}\right| .
$$

Here we consider a two-electron harmonium atom with $N=2$. The ground-state energy for a rigid potential diverges [32] at large $\omega$ as

$$
E(\omega) \simeq 3 \omega \quad(\omega \rightarrow \infty) .
$$

At a shallow harmonic potential, the energy can be expanded [32] in powers of $\omega$ giving

$$
E(\omega) \simeq \sum_{n=0}^{k} c_{n} \omega^{(2+n) / 3} \quad(\omega \rightarrow 0) .
$$


In low orders, one has

$$
E(\omega) \simeq c_{0} \omega^{2 / 3}+c_{1} \omega+c_{2} \omega^{4 / 3} \quad(\omega \rightarrow 0),
$$

with the coefficients

$$
c_{0}=\frac{3}{2^{4 / 3}}=1.19055, \quad c_{1}=\frac{1}{2}(3+\sqrt{3})=2.36603, \quad c_{2}=\frac{7}{36} 2^{-2 / 3}=0.122492 .
$$

Introducing the new variable

$$
x \equiv \omega^{1 / 3},
$$

equation (78) reduces to

$$
E\left(x^{3}\right) \simeq c_{0} x^{2}\left(1+a_{1} x+a_{2} x^{2}\right) \quad(x \rightarrow 0),
$$

with the coefficients

$$
a_{1}=\frac{c_{1}}{c_{0}}=1.98734, \quad a_{2}=\frac{c_{2}}{c_{0}}=0.102887 .
$$

Employing our method, we find the large $\omega$ behaviour

$$
E_{1}^{*}(\omega) \simeq 2.322 \omega^{\beta_{1}} \quad(\omega \rightarrow \infty)
$$

and

$$
E_{2}^{*}(\omega) \simeq 1.906 \omega^{\beta_{2}} \quad(\omega \rightarrow \infty)
$$

where

$$
\beta_{1}=1.018, \quad \beta_{2}=1.079 .
$$

The error of $\beta_{1}$ is $1.8 \%$ and of $\beta_{2}$, it is $7.9 \%$. The accuracy is compared with the known numerical data [33]. The resulting effective critical index at infinity is

$$
\beta^{*}=1.049 \pm 0.031 \text {. }
$$

The Dlog-Padé method, with the approximant $P_{1 / 1}$, has no physical solutions.

\section{Compressibility factor of hard-sphere fluids}

The state of hard-sphere fluids is described by the compressibility factor

$$
Z=\frac{P}{\rho k_{B} T}=Z(y) \quad\left(y \equiv \frac{\pi \rho}{6} a_{s}^{3}\right),
$$

in which $P$ is pressure, $\rho$ is density, $T$ is temperature, $a_{s}$ is the sphere diameter, and $y$ is called packing fraction [34].

The compressibility factor exhibits critical behaviour at a finite critical point. This behavior has been found from phenomenological equations [35] 38] as

$$
Z(y) \simeq 2\left(y_{c}-y\right)^{-t} \quad\left(y \rightarrow y_{c}-0\right),
$$

with the fitted parameters $y_{c}=1$ and $t=3$, although these are not asymptotically exact values.

For low packing fraction, the compressibility factor is represented by the virial expansion

$$
Z(y) \simeq 1+4 y+10 y^{2} .
$$

Using the method of Sec. 2 , we find, with $y_{c}=1$, the indices $t_{1}=4$ and $t_{2}=4.686$. Therefore our prediction for the critical index is

$$
t^{*}=4.343 \pm 0.34
$$

The Dlog-Padé method, with the approximant $P_{1 / 1}$, gives $t=4$. 


\section{Expansion factor of polymer chain}

The expansion factor of a polymer chain, as a function of the dimensionless coupling parameter $g$, can be expressed by the phenomenological equation [39, 40.

$$
\alpha(g)=\left(1+7.52 g+11.06 g^{2}\right)^{0.1772} .
$$

At large $g$, this gives

$$
\alpha(g) \simeq 1.531 g^{\beta} \quad(g \rightarrow \infty),
$$

with the critical index at infinity $\beta=0.3544$. One also considers the critical index

$$
\nu \equiv \frac{1}{2}\left(1+\frac{\beta}{2}\right)
$$

that here is $\nu=0.5886$. Other numerical calculations [41] give $\nu=0.5877$. At small $g$, perturbation theory yields [39,40] the expansion

$$
\alpha(g) \simeq 1+\frac{4}{3} g-2.075385 g^{2} \quad(g \rightarrow 0) .
$$

By the method of Sec. 2, we obtain the critical behaviour

$$
\alpha_{1}^{*}(g) \simeq 1.544 g^{\beta_{1}} \quad(g \rightarrow \infty)
$$

with the critical indices

$$
\beta_{1}=0.2999, \quad \nu_{1}=0.5750,
$$

the error being just $0.023 \%$. And the error of $\nu_{2}=0.5878$ is only $0.0013 \%$. In this way,

$$
\nu^{*}=0.5814 \pm 0.006
$$

The Dlog-Padé method, with the approximant $P_{1 / 1}$, does not possess physical solutions.

\section{Sedimentation coefficient of rigid spheres}

Sedimentation is a fundamental problem of studying how a suspension moves under gravity. The considered dispersion is build of small rigid spheres with random positions falling through Newtonian fluid under gravity. The mixture of solid particles and the fluid in a container is assumed to be homogeneous. The particles settle out under gravity at a rate depending, in particular, on concentration originating from hydrodynamic interactions between particles. The basic quantity of interest is the sedimentation velocity $U$, which is the averaged velocity of suspended particles, measured with respect to the velocity $U_{0}$ with which a single particle would move in the suspending fluid under the given force field in the absence of any other particles. This ratio is termed the collective mobility or sedimentation coefficient. The dependence of the collective mobility at low packing fractions is similar to the single-particle mobility, but quickly decreases at high packing fractions. The problem of sedimentation reminds that of Darcy flow in a porous medium, although their relation is not simple, because the physics of particle interactions is rather different. More details on the physics of sedimentation can be found in the paper by Batchelor [42]. 
The dimensionless sedimentation velocity $u \equiv U / U_{0}$ is considered as a function of the packing fraction $f$. This velocity exhibits the critical behaviour

$$
u(f) \propto(1-f)^{\beta} \quad(f \rightarrow 1-0)
$$

at the critical point $f_{c}=1$. The critical index, however, has been defined differently by different authors. Thus Batchelor [42] gives $\beta=5$. While other authors [43 46] suggest $\beta=3$. Below we find the critical index being based on the expansion derived by Cichocki et al [47],

$$
u(f) \simeq 1-6.546 f+21.918 f^{2} \quad(f \rightarrow 0) .
$$

By the method of Sec. 2 , setting $f_{c}=1$, we find $\beta_{1}=3.0438$ and $\beta_{2}=3.5660$. Therefore we predict the critical index

$$
\beta^{*}=3.3049 \pm 0.26
$$

The Dlog-Padé method, with the approximant $P_{1 / 1}$, has no physical solutions.

\section{Ground-state energy of Schwinger model}

The Schwinger model [48,49] represents Euclidean quantum electrodynamics with a Dirac fermion field, which is formulated as a lattice gauge theory in $(1+1)$ dimensions. It enjoys many properties in common with quantum chromodynamics, such as confinement, chiral symmetry breaking, and charge shielding, because of which it has become a standard test bed for the study of numerical techniques. Here we consider the model corresponding to a vector boson of mass $M(x)$ as a function of the dimensionless variable $x \equiv m / g$, with $m$ being the electron mass and $g$ being the coupling parameter. The ground-state energy is given by the expression $E-2 m$.

The energy, as a function of $x$, increases with $x$, reaching the asymptotic value [50-53]

$$
E(x) \simeq 0.6418 x^{\beta} \quad(x \rightarrow \infty),
$$

with the critical index at infinity $\beta=1 / 3$.

At small $x$, there is the expansion [50,54,56]

$$
E(x) \simeq 0.5642\left(1-0.38816 x+0.338001 x^{2}\right) \quad(x \rightarrow 0) .
$$

Using the method of Sec. 2 , we find $\beta_{1}=-0.2868$ that differs from the exact $\beta=-1 / 3$ by $13.96 \%$ and $\beta_{2}=-0.3360$ differing from the exact value by $0.8 \%$. Thus, we get

$$
\beta^{*}=0.311 \pm 0.02 .
$$

The Dlog-Padé method, with the approximant $P_{1 / 1}$, does not have physical solutions.

\section{Convergence of approximants for critical indices}

In the present section, we illustrate the numerical convergence of root approximants applied for calculating critical indices. As an example, we consider the pressure $P(x)$ of a fluctuating fluid membrane [57] as a function of stiffness $x$. This example is of special interest, since it cannot be treated by Padé approximants [12]. 
The pressure can be represented in the form

$$
P(x)=\frac{\pi^{2}}{8 x^{2}} f(x)
$$

It has been found by Monte Carlo simulations [58] that the function $f(x)$ diverges at infinity as

$$
f(x) \simeq 0.06468 x^{2} \quad(x \rightarrow \infty) .
$$

Hence, this function exhibits the critical behaviour at infinity with the critical index $\beta=2$.

At weak stiffness, the function $f(x)$ can be found [59] by perturbation theory with respect to the stiffness, yielding the expansion

$$
f(x) \simeq \sum_{n=0}^{k} a_{n} x^{n} \quad(x \rightarrow 0)
$$

with the coefficients

$$
\begin{gathered}
a_{0}=1, \quad a_{1}=\frac{1}{4}, \quad a_{2}=\frac{1}{32}, \quad a_{3}=2.176347 \times 10^{-3}, \\
a_{4}=0.552721 \times 10^{-4}, \quad a_{5}=-0.721482 \times 10^{-5}, \quad a_{6}=-1.777848 \times 10^{-6},
\end{gathered}
$$

which can be complemented by two more coefficients $a_{7}=a_{8}=0$.

We construct the root approximants

$$
f_{k}^{*}(x)=\left(\left(\left(\left(1+A_{1} x\right)^{2}+A_{2} x^{2}\right)^{3 / 2}+A_{3} x^{3}\right)^{4 / 3}+\ldots+A_{k} x^{k}\right)^{\beta_{k} / k}
$$

defining the parameters $A_{j}$ from the accuracy-through-order procedure, as is explained in Sec. 1. This gives the large-stiffness asymptotic forms

$$
f_{k}^{*}(x) \simeq B_{k} x_{k}^{\beta} \quad(x \rightarrow \infty),
$$

where the amplitudes $B_{k}=B_{k}\left(\beta_{k}\right)$ are

$$
B_{k}=\left(\left(\left(A_{1}^{2}+A_{2}\right)^{3 / 2}+A_{3}\right)^{4 / 3}+\ldots+A_{k}\right)^{\beta_{k} / k}
$$

In order to define the critical index $\beta_{k}$, we follow Sec. 2 and analyze the differences

$$
\Delta_{k n}\left(\beta_{k}\right)=B_{k}\left(\beta_{k}\right)-B_{n}\left(\beta_{k}\right) .
$$

Composing the sequences $\Delta_{k n}=0$, we find the related approximate values $\beta_{k}$ for the critical indices. It is possible to investigate different sequences of the conditions $\Delta_{k n}=0$, the most logical from which are the sequences of $\Delta_{k, k+1}=0$ and of $\Delta_{k 8}=0$, with $k=1,2,3,4,5,6,7$. The results, presented in Table 1 , show good numerical convergence of the approximate critical indices $\beta_{k}$ to the exact value $\beta=2$.

The Dlog-Padé method, with the approximant $P_{1 / 1}$, again does not provide physically acceptable solutions. And if we employ the approximant $P_{N / N+1}$, this results in the sequence of the critical indices $-0.08095,2.5188,-5.3603$, and -2.19958 for $N=1,2,3,4$, respectively. As is evident, such a sequence is neither convergent nor reasonable. 


\begin{tabular}{|c|c|c|}
\hline$\beta_{k}$ & $\Delta_{k, k+1}\left(\beta_{k}\right)=0$ & $\Delta_{k 8}\left(\beta_{k}\right)=0$ \\
\hline$\beta_{1}$ & 24.9036 & 2.5052 \\
\hline$\beta_{2}$ & 4.9344 & 2.4701 \\
\hline$\beta_{3}$ & 3.4791 & 2.3887 \\
\hline$\beta_{4}$ & 2.8459 & 2.2970 \\
\hline$\beta_{5}$ & 2.3983 & 2.2018 \\
\hline$\beta_{6}$ & 2.2040 & 2.1289 \\
\hline$\beta_{7}$ & 2.0645 & 2.0645 \\
\hline
\end{tabular}

Table 1: Critical indices $\beta_{k}$ for the problem of Sec. 14, obtained from the optimization conditions $\Delta_{k n}\left(\beta_{k}\right)=0$. The sequences of $\beta_{k}$ demonstrate numerical convergence to the exact value $\beta=2$.

\section{Concluding comments}

The method of self-similar root approximants, proved earlier to provide accurate interpolation for the sought function, when both the asymptotic expansions at small and large variables are known. Now the method is generalized to the case when only a small-variable expansion is available and the function can display critical behaviour at a finite critical point or at infinity. We show how, having in hands only a small-variable expansion, one can construct extrapolation formulas for the sought function and to find its critical index. The method of defining critical indices is illustrated by a large set of examples for various physical problems. It is shown that the suggested approach makes it straightforward to calculate critical indices, with a good accuracy, even when just a few terms of small-variable expansions are available. When a number of terms in the small-variable expansion is given, the method demonstrates numerical convergence to the exact indices, if the latter are known.

Following the idea of the approach, it is admissible to realize different variants of the calculational scheme. For instance, instead of treating the sought function $f(x)$, and its related $k$-order expansion $f_{k}(x)$, one can consider the inverse expression $h_{k}(x) \equiv 1 / f_{k}(x)$. Then, constructing the root approximants $h_{k}^{*}(x)$, it is easy to return back to $f_{k}^{*}(x)=1 / h_{k}^{*}(x)$.

The other possibility can be useful, when the critical behaviour occurs at a finite critical point $x_{c}$. By the change of the variable

$$
z=\frac{x}{x_{c}-x}, \quad x=\frac{x_{c} z}{1+z}
$$

one shifts the critical point to infinity. And then finds the critical index as is explained in Sec. 2 .

We have checked both these variants for the examples considered and found that they give close results for the critical indices, as compared to the direct method exposed above.

In conclusion, the developed method of defining critical indices is general and can be applied to different physical problems. The method works well even when other methods, such as that of Padé approximants, are not applicable.

\section{Acknowledgment}

One of the authors (V.I.Y.) is grateful for discussions to E.P. Yukalova. 


\section{References}

[1] G.A. Baker, P. Graves-Moris, Padé Approximants (Cambridge University, Cambridge, 1996).

[2] H. Kleinert, Path Integrals in Quantum Mechanics, Statistics, Polymer Physics and Financial Markets (World Scientific, Singapore, 2006).

[3] S. Gluzman, V.I. Yukalov, Eur. J. Appl. Math. 25, 595 (2014).

[4] S. Gluzman, V.I. Yukalov, Eur. Phys. J. Plus 131, 340 (2016).

[5] V.I. Yukalov, Phys. Rev. 42, 3324 (1990).

[6] V.I. Yukalov, J. Math. Phys. 32, 1235 (1991).

[7] V.I. Yukalov, J. Math. Phys. 33, 3994 (1992).

[8] V.I. Yukalov, S. Gluzman, Phys. Rev. Lett. 79, 333 (1997).

[9] S. Gluzman, V.I. Yukalov, Phys. Rev. E 58, 4197 (1998).

[10] V.I. Yukalov, E.P. Yukalova, S. Gluzman, Phys. Rev. A 58, 96 (1998).

[11] V.I Yukalov, E.P. Yukalova, S. Gluzman, J. Math. Chem. 47, 959 (2010).

[12] S. Gluzman, V.I. Yukalov, J. Math. Chem. 48, 883 (2010).

[13] S. Gluzman, V.I. Yukalov, Mathematics 3, 510 (2015).

[14] V.I. Yukalov, S. Gluzman, Phys. Rev. D 91, 125023 (2015).

[15] V.L. Kalitvyansky, A.P. Kashin, M.Z. Maximov, Z.E. Chikovani, J. Nucl. Phys. 41, 329 (1985).

[16] H.X. He, C.J. Hamer, J Oitmaa, J. Phys. A 23, 1775 (1990).

[17] R.J. Baxter, Exactly Solved Models in Statistical Mechanics (Academic, London, 1982).

[18] P. Butera, M. Comi, J. Stat. Phys. 109, 311 (2002).

[19] H. Kleinert, V. Schulte-Frohlinde, Critical Properties of $\varphi^{4}$ - Theories (World Scientific, Singapore, 2006).

[20] G.K. Batchelor, J.T. Green, J. Fluid Mech. 56, 401 (1972).

[21] E. Wajnryb, J.S. Dahler, Adv. Chem. Phys. 102, 193 (1997).

[22] B. Cichocki, M.L. Ekiel-Jezewska, E. Wajnryb, J. Chem. Phys. 119, 606 (2003).

[23] D. Stauffer, A. Coniglio, M. Adam, Adv. Polymer Sci. 44, 103 (1982).

[24] T.M. Nieuwenhuizen, P.F.J. van Velthoven, M.H. Ernst, Phys. Rev. Lett. 57, 2477 (1986).

[25] D. Frenkel, Phys. Lett. A 121, 385 (1987). 
[26] P. Grassberger, Physica A 262, 251 (1999).

[27] S. Kirkpatrick, Rev. Mod. Phys. 45, 574 (1973).

[28] F. Hofling, T. Franosch, E. Frey, Phys. Rev. Lett. 96, 165901 (2006).

[29] T. Bauer, F. Hofling, T. Munk, E. Frey, T. Franosch, Eur. Phys. J. Spec. Top. 189, 103 (2010).

[30] P.M. Adler, Porous Media. Geometry and Transport (Batterworth-Heinemann, Oxford, 1992).

[31] A.E. Malevich, V.V. Mityushev, P.M. Adler, Acta Mech. 182, 151 (2006).

[32] J. Cioslowski, J. Chem. Phys. 136, 044109 (2012).

[33] E. Matito, J. Cioslowski, S.F. Vyboishchikov, Phys. Chem. Chem. Phys. 12, 6712 (2010).

[34] A. Mulero, Theory and Simulation of Hard-Sphere Fluids and Related Systems (Springer, Berlin, 2008).

[35] G.W. Wu, R.J. Sadus, Am. Inst. Chem. Eng. J. 51, 309 (2005).

[36] J. Tian, H. Jiang, Y. Gui, A. Mulero, Phys. Chem. Chem. Phys. 11, 11213 (2009).

[37] J. Tian, Y. Gui, A. Mulero, J. Phys. Chem. B 114, 13399 (2010).

[38] X.Z. Wang, H.R. Ma, Chin. J. Chem. Phys. 23, 675 (2010).

[39] M. Muthukumar, B.G. Nickel, J. Chem. Phys. 80, 5839 (1984).

[40] M. Muthukumar, B.G. Nickel, J. Chem. Phys. 86, 460 (1987).

[41] B. Li, N. Madras, A.D. Sokal, J. Stat. Phys. 80, 661 (1995).

[42] G.K. Batchelor, J. Fluid Mech. 52, 245 (1972).

[43] C.W.J. Beenakker, P. Mazur, Physica A 120, 388 (1984).

[44] J.F. Brady, L.J. Durlofsky, Phys. Fluids 31, 717 (1988).

[45] A.J.C. Ladd, J. Chem. Phys. 93, 3484 (1990).

[46] H. Hayakawa, K. Ichiki, Phys. Rev. E 51, 3815 (1995).

[47] B. Cichocki, M.L. Ekiel-Jezewska, P. Szymczak, E. Wajnryb, J. Chem. Phys. 117, 1231 (2002).

[48] J. Schwinger, Phys. Rev. 128, 2425 (1962).

[49] T. Banks, L. Susskind, J. Kogut, Phys. Rev. D 13, 1043 (1976).

[50] P. Striganesh, C. J. Hamer, R. J. Bursill, Phys. Rev. D 62, 034508 (2000).

[51] S. Coleman, Ann. Phys. (N.Y.) 101, 239 (1976). 
[52] C. J. Hamer, Nucl. Phys. B 121, 159 (1977).

[53] C. J. Hamer, Z. Weihong, J. Oitmaa, Phys. Rev. D 56, 55 (1997).

[54] A. Carrol, J. Kogut, D. K. Sinclair, L. Susskind, Phys. Rev. D 13, 2270 (1976).

[55] J. P. Vary, T. J. Fields, H. J. Pirner, Phys. Rev. D 53, 7231 (1996).

[56] C. Adam, Phys. Lett. B 382, 383 (1996).

[57] U. Seifert, Adv. Phys 46, 13 (1997).

[58] G. Gompper, D.M. Kroll, Eur. Phys. Lett. 9, 59 (1989).

[59] B. Kastening, Phys. Rev. E 73, 011101 (2006). 